\title{
Inbreeding depression in fecundity and inbred line extinction in the bulb mite, Rhizoglyphus robini
}

\author{
J Radwan ${ }^{1,2}$ \\ ${ }^{1}$ Institute of Environmental Sciences, Jagiellonian University, ul. Ingardena 6, Cracow, Poland; ${ }^{2}$ Department of Biology, University of \\ New Mexico, Albuquerque, NM 87131, USA
}

\begin{abstract}
This study investigated the magnitude of inbreeding depression in fecundity, and whether the depression is purged during six generations of sib mating in the bulb mite, Rhizoglyphus robini. The progeny resulting from a single generation of brother-sister mating suffered significant inbreeding depression in fecundity. During the following six generations of continuous sib-mating, 58\% lines were lost, $45 \%$ because of sterility and $13 \%$ because of preadult mortality. The lines were then outcrossed, and their inbreeding depression compared with that of the base population. The inbreeding depression for the outcrossed population was 0.15 , and for the base population 0.19 , but the difference was not significant. The lack of significant purging of inbreeding depression indicates that it was caused either by detrimental genes of small effect, or by the breaking down of over-
\end{abstract}

dominant relations between alleles. However, the large proportion of extinct lines points to the former mechanism as a predominant cause of inbreeding depression. Theory predicts that the probability of line extinction with inbreeding increases with its load of mutations. If phenotypic variation in fecundity was partly because of differences in numbers of mutations carried by individuals, the fecundity of the line founder could be expected to correlate with the probability that the line derived from it will survive long-term inbreeding. Indeed, fecundity of founder females was significantly associated with line survival, which suggests that line extinction rate may be used as a method to study individual mutational loads, for example, in studies of sexual selection. Heredity (2003) 90, 371-376. doi:10.1038/sj.hdy.6800254

Keywords: genetic load; mutations; purging; fitness; sexual selection

\section{Introduction}

Inbreeding depression, the decline in trait value as a result of inbreeding, has been reported from many animal and plant species (reviewed by Charlesworth and Charlesworth, 1987, 1999). Among the important implications of this phenomenon is the decline in fitness observed in small populations, a phenomenon of major concern in conservation biology (Frankham, 1995; Keller and Waller, 2002). Most of the evidence accumulated so far points to partially recessive deleterious mutations as a main source of inbreeding depression (Charlesworth and Charlesworth, 1999; Crow, 1999), although overdominance can also play an important role in some organisms (eg Karkkainen et al, 1999). Tempelton and Read (1983) suggested that inbreeding depression can be purged from endangered populations through controlled inbreeding, which increases the effectiveness of selection against deleterious recessives. As no purging occurs under overdominance, crossing inbred lines should restore fitness to the original value, whereas if purging of deleterious alleles occurred during inbreeding, fitness is expected to be higher in the outcrossed lines than in the base population (Barrett and Charlesworth, 1991; Roff, 2002). However, Willis (1999) pointed out that

Correspondence: J Radwan, Institute of Environmental Sciences, Jagiellonian University, ul. Ingardena 6, Cracow, Poland.

E-mail: radwan@eko.uj.edu.pl

Received 17 May 2002; accepted 29 January 2003 evolution in the original population or in the inbred lines may invalidate conclusions based on this prediction. He proposed that the decrease in inbreeding depression, rather than the increase in fitness in populations with a history of inbreeding, should be demonstrated to imply purging (Figure 1 in Willis, 1999). The present study applies the design of Willis (1999) to test for purging of inbreeding depression in female fecundity in the bulb mite, Rhizoglyphus robini (Acari: Acaridae).

The effectiveness of purging will differ depending on inbreeding rate or breeding scheme, and, importantly, on the type of the underlying mutations (Hedrick, 1994, Wang et al, 1999; Wang, 2000). Lethal and sublethal mutations can easily be eliminated from inbred lines, but mutations that are only weakly selected against (ie detrimentals) will often be fixed in populations via a random drift, and purging will be much less effective (Hedrick, 1994). Moreover, these detrimental mutations tend to be only partially recessive, so inbreeding does not increase the selection against them as much as against more recessive lethals or sublethals (Hedrick, 1994; Wang et al, 1999). Thus, the degree to which inbreeding depression is purged can be used to infer whether it is caused by genes of large or small effects (Willis, 1999): if inbreeding depression is caused mainly by detrimentals, purging will be much slower than for lethals (Hedrick, 1994). In practice, it might be difficult to determine whether inbreeding depression is caused by overdominance or recessive detrimentals when no significant purging is observed. However, the latter, but not the 
former, mechanism predicts the extinction of inbred lines in which deleterious mutations become fixed (Hedrick, 1994).

The extinction of a large proportion of lines is a typical result of several generations of brother-sister mating (eg Bowman and Falconer, 1960; Sittman et al, 1966). Hedrick (1994) showed that, among other factors, the proportion of lines lost during inbreeding depends on the number of lethal equivalents present within a line. Inversely, the chance of line extinction could therefore be used as a measure of genetic load within the line. In this study, two inbred lines were derived from each founder female whose fecundity was measured. If phenotypic variation in fecundity was partly caused by deleterious mutations, I expected a negative correlation between fecundity of founders and the probability of extinction of the lines derived from them.

\section{Methods}

Rhizoglyphus robini infests subterranean structures of plants, for example, bulbs of onions, garlic and other members of the Liliaceae. It also infests stored food products (Diaz et al, 2000). Like other acarid mites, Rhizoglyphus is diploid and lacks parthenogenesis (Oliver, 1971, 1977). Sex ratio at emergence of adults is near unity (Gerson et al, 1983). Little is known about the population structure of the species. It occurs in populations ranging from a few to hundreds of individuals (reviewed in Diaz et al, 2000), so populations may undergo occasional bottlenecks. The mites used in this study came from a stock culture derived from a colony of about 100 individuals found on onions from a garden near Cracow, Poland, in 1998. They were kept in the lab as a large population (>1000 individuals) for 2 years (about 50 generations) before the commencement of this research. The culture was maintained at $22-26^{\circ} \mathrm{C},>90 \%$ humidity, and fed a 3:1 mixture of powdered yeast and wheat germ.

During experiments, individual and paired mites were kept in $50 \mathrm{ml}$ Eppendorf vials filled to $1 / 3$ height with solidified, moist plaster-of-paris darkened with $10 \%$ powdered charcoal for better visualisation of the whitish-coloured mites on the background. Vials were closed with nonabsorbent cotton wool. Food was provided ad libitum and temperature maintained at $22^{\circ} \mathrm{C}$.

Virgin females, obtained from individually isolated larvae, were randomly mated with a single male, and six eggs were isolated from each such family. Upon reaching adulthood, the virgin female offspring of these pairs were mated either to their brothers, or to a randomly assigned, unrelated male. Those mated to their brothers remained paired with them for 3 days, and the eggs laid during this period were used to start the next inbred generation. After 3 days, the brothers were removed and the females paired with a second, this time unrelated, male. The eggs laid later, fertilised by an unrelated male (as a result of the last male sperm precedence, Radwan, 1997) were used only for fecundity assessment and then discarded. Fecundity was assessed by counting the eggs laid on the 6 days following mating with an unrelated male. This period is fairly representative of a female's lifetime egg output, as female oviposition rate does not change significantly over her egg-laying period of about 3 weeks, (Konior et al, 2001).

Outbred pairs were less numerous than inbred pairs because not all pairs produced two progeny of each sex, and the number of inbreds was maximised for the purpose of the long-term inbreeding experiment. In total, 16 eggs were collected from inbred and outbred females and left to develop. Upon reaching adulthood, two females from each family (inbred as well as outbred) were mated to an unrelated male and their fecundity assessed as in the previous generation. Additionally, two other females from each inbred family were mated to their brothers in order to start inbred lines. For the consecutive five generations, two lines from each of the $155 \mathrm{~F}_{1}$ brother-sister founding pairs (ie a total of 310 lines) were propagated through sib-mating: 10 eggs were isolated from each pair, and upon reaching adulthood, one pair was randomly selected for mating. Lines could go extinct because no individuals reached adulthood (egg/juvenile mortality) or because the pair produced no eggs (sterility). Females do not lay eggs unless they are fertilised, and stop ovipositing when sperm is depleted (Woodring, 1969; Radwan, 1997). This is probably because, in acarid mites, sperm penetration into oocytes is necessary for egg development to proceed beyond the diplotene (Heinemann and Hughes, 1970). Thus, all unhatched eggs were due to embryonic mortality and not because they were not fertilised. A small proportion of families (not different from the expectation of $1 / 2^{9}$ per generation) was lost because all the progeny isolated were of the same sex. Lines lost for this reason were not entered into further analysis.

In the sixth inbred generation, one female from each surviving line (one per founding pair where two lines survived) was mated to an unrelated male to produce $F_{7}$ outcrosses. At the same time, females, isolated from the base colony as larvae 8 days earlier and kept under identical conditions as inbred $\mathrm{F}_{6}$ to minimise maternal effects, were each mated to a random male. Upon reaching adulthood, one female from each of the $F_{7}$ outcrossed families, as well as from the simultaneously maintained base population families, was mated to their brother, and another female to an unrelated male. Where only one female was obtained from a family, it was assigned randomly to the inbred or outbred group. Their progeny were reared individually in the same desiccator, and upon reaching adulthood, two females from each inbred and outbred families were mated to a random male and their fecundity estimated for 6 days following mating.

Inbreeding depression was calculated as $1-\hat{w}_{\mathrm{i}} / \hat{w}_{\mathrm{O}}$, where $\hat{w}_{\mathrm{i}}$ is a mean trait value for inbred population, $\hat{w}_{\mathrm{O}}$ for outbred population. The significance of inbreeding depression was tested with the $t$-statistic calculated according to Lynch and Walsh (1998), as $t=\left(\hat{w}_{\mathrm{i}}-\hat{w}_{\mathrm{o}}\right) /\left(\mathrm{SD}_{\mathrm{o}}(2 / L)^{1 / 2}\right)$, where $\mathrm{SD}_{\mathrm{o}}$ is the standard deviation of the trait measured in outbreds and $L$ the number of families. This method takes into account that the variance is expected to be higher among inbreds.

The difference in the magnitude of inbreeding depression between outcrossed $\mathrm{F}_{7}$ and the base population was first tested by calculating differences between means for inbreds and outbreds from each maternal family (Willis, 1999). As expected, these differences were normally distributed around the mean of zero $(P>0.2$ for both 
base and outcrossed $\mathrm{F}_{7}$, Kolmogorov-Smirnoff test). However, as 21 families had no maternal equivalent (because of random events or the lack of the second female within the family in the previous generation), inbreeding depression was also compared from mean fecundities of outcrossed $\mathrm{F}_{7}$ and base populations, which allowed inclusion of all data. This was analysed using standard ANOVA, as it is robust against moderate departures from heteroscedasticity (Glass et al, 1972), and Bartlett's test did not detect significant heterogeneity of variances in the data $(P=0.84)$. In this analysis, purging of inbreeding depression would be detected as a significant interaction between source population (base or outcrossed) and treatment (inbred or outbred).

As variances in fecundity between lines that lost zero, one and two lines during six generations of inbreeding differed substantially ( $P=0.014$, Bartlett's test), nonparametric tests were applied.

\section{Results}

The first analysis of female fecundity was performed at the beginning of the experiment. It revealed significant inbreeding depression: outbred females from the second generation laid on average $( \pm S D)$ 97.4 \pm 26.1 eggs $(n=88)$, and same-generation inbred females (inbreeding coefficient $\mathrm{F}=0.25)$ laid $76.1 \pm 31.3$ eggs $(n=162 ; t=7,29$, $P<0.001$ ). Inbreeding depression was thus 0.22 , and a linear extrapolation for the completely inbred population $(\mathrm{F}=1)$ yielded an inbreeding depression coefficient $(\delta)$ of 0.87 .

During the following six generations of brother-sister mating, $58 \%$ of lines were lost, $13 \%$ because of pre-adult (mostly embryonic) mortality, and 45\% because of sterility (lines lost because of chance events or samesex progenies are not included). That such a high extinction rate was because of inbreeding and not residual sterility is indicated by the fact that the proportion of outbred pairs that did not produce any eggs was very low $(0.9 \%, n=88$ outbred pairs from the second generation), as was the proportion of pairs that failed to produce viable progeny $(1.3 \%)$. Thus, the expected line extinction for both the reasons was $2.2 \%$ per generation, which, over six generations, should yield a cumulative effect of $12.5 \%$ lines lost, significantly less than actually recorded $\left(\chi^{2}=28.5, \mathrm{df}=1, P<0.001\right.$, $n=288)$.

Lines could also be lost if there were strong barriers against sib-mating. However, the proportion of sibmated females that did not produce any eggs was very low $(0.9 \%, n=170$ inbred pairs from the second generation) and identical to that for the same generation females mated to unrelated males (above). Thus the line extinctions that occurred are likely to be the result of the fixation of deleterious alleles.

The deleterious alleles that caused line extinction may have also been important in causing variation in fecundity among founder females. Mean fecundity would therefore be expected to be the highest in the group in which two lines survived, and the lowest in the group with no surviving lines. This prediction was tested with a directional heterogeneity test (Rice and Gaines, 1994). No a priori predictions could be made as to which cause of extinction (sterility or inviability) is more likely to correlate with the founder fecundity, so all cases of extinction were pooled. The means \pm SDs for founder females that gave rise to two, one and zero surviving lines were, respectively: $115.5 \pm 19.3(n=25), 107.3 \pm 32.5$ $(n=52)$ and $102.9 \pm 31.7(n=54)$, that is, they were ordered in the expected direction $\left(r_{\mathrm{s}}=1\right)$. The directional heterogeneity test, based here on a nondirectional $P$ value obtained from a Kruskal-Wallis test $(H=4.55$, $n=131, P=0.103)$, revealed that the ordering of the means was significant $\left(r_{\mathrm{s}} P_{\mathrm{c}}=0.87, P<0.02\right)$. Similar analyses conducted separately for each cause of extinction showed the same ordering of the means, and were both significant by the directional heterogeneity tests.

The preceding analyses only included founding females for which none of the lines were lost through chance events, that is about $80 \%$ of the original number. Another prediction is that founder female fecundity should correlate positively with the average number of generations survived by brother-sister lines derived from them (Radwan, in preparation). This prediction, tested after inclusion of lines whose second replicates were lost because of chance events, was also borne out (Figure 1, $r_{\mathrm{s}}=0.17, n=155, P=0.035$ ).

The mean fecundity differences $( \pm S D)$ between outbreds and inbreds in maternal families were $15.9 \pm 50.1$, $n=22$ for outcrossed $\mathrm{F}_{7}$, and $19.5 \pm 47.5, n=26$ for base populations, and there was no evidence for purging $\left(t_{56}=-0.34, P=0.73\right)$. Analysis of the extended data set (ie after inclusion of the families that had no maternal counterpart among inbreds/outbreds) with ANOVA yielded the same conclusion: the interaction between the effects of population (outcrossed $\mathrm{F}_{7}$ or base) and inbreeding, which would be indicative of purging, was not significant (Table 1). There was a significant effect of inbreeding, but not of the source population (Table 1). Mean fecundities of inbreds and outbreds from base and outcrossed $\mathrm{F}_{7}$ populations are shown in Figure 2. Inbreeding depression for $\mathrm{F}=0.25$ in the base population was 0.19 , and in $\mathrm{F}_{7}$ outcrossed population 0.15 .

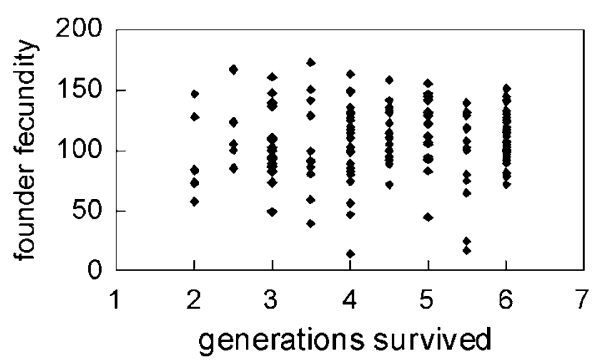

Figure 1 The relation between female fecundity and mean number of generations survived by the inbred lines derived from them.

Table 1 Results of analysis of variance with egg number laid during 6 days as a dependent variable, and source population (base or outcrossed inbred lines) and inbreeding as fixed factors

\begin{tabular}{lrrrr}
\hline Source & $d f$ & MS & $F$ & $\mathrm{P}$ \\
\hline Source population & 1 & 2276.8 & 2.444 & 0.120 \\
Inbreeding & 1 & 8686.1 & 7.644 & 0.006 \\
Interaction & 1 & 330.4 & 0.291 & 0.591 \\
Error & 140 & 1136.4 & & \\
\hline
\end{tabular}




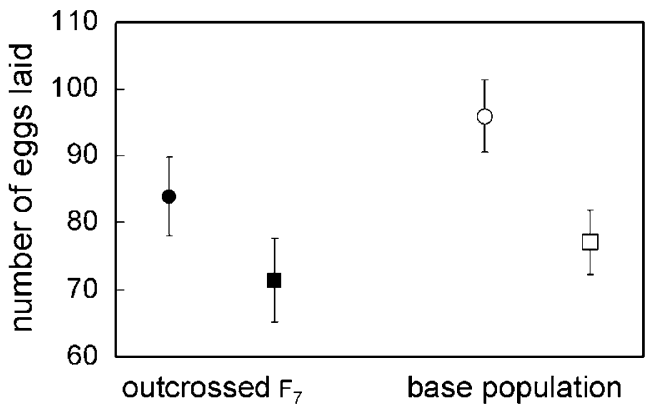

Figure 2 Fecundities (mean \pm standard error) of inbred $(F=0.25$, squares) and outbred (circles) females whose parents were obtained by outcrossing inbred lines (filled symbols) or came from the base population (empty symbols).

\section{Discussion}

This study shows that bulb mite females suffer substantial inbreeding depression in fecundity; the two estimates for the base population, one obtained at the beginning of the experiment, and the second simultaneously with the outcrossed $\mathrm{F}_{7}$ generation, yielded similar values of the inbreeding depression coefficient ( 0.22 and 0.19 for $\mathrm{F}=0.25$ ). However, no evidence for purging of inbreeding depression was found. The inbreeding depression coefficient in outbreds created by the crossing of inbred lines was 0.15 , but this was not significantly less than in the base population. Even if the lack of significance resulted from insufficient statistical power, this result shows that purging was at best very modest. Comparison of the fecundity differences between inbreds and outbreds within maternal families also failed to find evidence for significant purging.

Hedrick (1994) simulated the process of purging under brother-sister mating with a family size of 10 , a design followed exactly in the present study. In Hedrick's (1994) simulations, lethals were purged from the population much faster than detrimentals irrespective of whether they caused sterility or mortality, even though both lethals and detrimentals caused substantial line extinction. For example, after six generations of sib mating less than a half of the initial load of two lethal equivalents was retained when the load was distributed between two lethals, whereas for detrimentals (selection coefficient 0.125 , ie two lethal equivalents distributed over 32 loci) less than $15 \%$ of the initial load was purged (Figures 5 and 6 in Hedrick, 1994). The lack of significant purging found in the present study thus indicates that inbreeding depression in the bulb mite is caused mainly by genes of small effects, which are unlikely to be purged during inbreeding (Hedrick, 1994; Wang et al, 1999), or that overdominance plays a substantial role. The high proportion of lines lost during inbreeding suggests the role of deleterious mutations: the breakdown of overdominant relations between alleles is expected to cause decreased fitness, but there is no reason to expect mortality or sterility. On the other hand, line extinction may occur even if the effects of detrimental mutations are additive (Hedrick, 1994) when such mutations are fixed in inbred lines. In this situation, with no dominance, no inbreeding depression occurs (Lynch and
Walsh, 1998; Roff, 1998). In theory, therefore, genes causing inbreeding depression may not be the same as genes causing line extinction, so that the role of overdominance in causing the former cannot be ruled out even when extinction is substantial. However, because deleterious mutations are usually at least partially recessive (Houle et al, 1997), inbreeding depression and line extinction are likely to be caused by the same genes.

Using the same design as in the present study, Willis (1999) found no evidence of purging in Mimulus guttatus. Purging may be more effective under slow inbreeding and large population size (Wang et al, 1999), and Fowler and Whitlock (1999) did find evidence for purging in Drosophila melanogaster under slow inbreeding. Unfortunately, such a design would not be practical for a deliberate purging programme, for example in endangered species, because of the many generations needed. Nevertheless, most of the evidence collected so far suggests that the purging process does not play a substantial role in the majority of populations. Ballou (1997) found little evidence for purging in 25 mammal populations, and a meta-analysis in plants (Byers and Waller, 1999) revealed that if purging occurs at all, its effects are usually in order of less than $10 \%$.

Several studies report that outbreds created from inbred lines had higher fitness than outbreds from populations that were not previously inbred (eg Lynch, 1977; Bryant et al, 1990; Barrett and Charlesworth, 1991; Garcia et al, 1994; Latter et al, 1995; Roff, 2002), which was taken as the evidence for purging. Willis (1999) found a similar effect in his study of $M$. guttatus, but he pointed out that the effect is likely to be caused by adaptation to laboratory conditions that had occurred in inbred lines. In the present study, no increase in fitness in the outcrossed $\mathrm{F}_{7}$ was found. However, as both the base population and inbred lines were kept in the laboratory, adaptation to lab conditions was not expected to be higher in inbreds. On the other hand, the lack of difference cannot be taken as the evidence against purging, as evolution in the base population cannot be excluded. The base population might have evolved a higher fitness than inbred lines, where selection was limited. Moreover, when all but viability and sterility selection is eliminated (as under inbreeding scheme used in the present study), fitness may decline at a rate as high as $2 \%$ per generation because of accumulation of deleterious mutations (Shabalina et al, 1997). This process could further decrease the fitness of inbred lines.

Hedrick (1994) suggested that extinction patterns of inbred lines can be used to infer the type of genetic load responsible for extinction. Viability lethals should not cause much extinction, as they should be effectively purged via selection within families, and line loss due to sterility lethals should be concentrated in a few early generations. On the other hand, detrimentals are expected to cause a much higher extinction rate because of inviability, and the extinction because of sterility should continue to increase with each generation of inbreeding (Hedrick, 1994). However, to discern these differences, about 20 generations of inbreeding would be needed. Moreover, Hedrick (1994) did not simulate joint effects of line sterility and inviability. In this study, 77\% of all extinctions were because of sterility; simulations 
allowing both causes of line extinction to operate simultaneously (Radwan, in preparation) suggest that such a situation (ie most extinctions due to line sterility) will occur when the number of loci for sterility and for viability is similar (assuming the same average selection coefficient per locus for both traits). For the extinction rates for both causes to be more equal, the number of loci affecting viability needs to be a few times larger than the number of fertility loci. This is because under a design similar to that used in the present study, selection against steriles can only operate via line extinction, and not via within-family selection.

Another potential application of the analysis of line extinction is in the assessment of individual mutational loads. The number of deleterious mutations carried by an individual will influence the probability of extinction of inbred lines derived from it (Hedrick, 1994). This will not only be true for recessive genes that cause inbreeding depression, but also for completely additive genes (Hedrick, 1994). More modelling and experimental effort is needed to determine the predictive power of this association. The data collected in the present study are encouraging. There was a significant negative association between the number of lines extinct and the fecundity of females from which the lines were derived. In Drosophila female, fecundity seems to represent a large mutational target (Houle, 1998). In $R$. robini, heritability of fecundity was estimated at $27 \%$ (Radwan et al, unpublished manuscript), so it seems plausible that in this species also variation in fecundity is caused, to a significant extent, by deleterious mutations, and that higher rates of extinction reflected higher mutation loads of founder females. A similar design could be used to test hypotheses concerning genetic quality of individuals, such as recent 'good genes' models of sexual selection that assume genetic variance in male quality to be caused by deleterious mutations (Houle and Kondrashov, 2002)

\section{Acknowledgements}

I thank $\mathrm{P}$ Watson and $\mathrm{R}$ Thornhill for many inspiring discussions, encouragement and logistic support, and J Tomkins, N LeBas and Z Prokop for their comments on previous versions of the manuscript. During the part of this study carried out at UNM, I was supported by the Fulbright Program.

\section{References}

Ballou JD (1997). Ancestral inbreeding only minimally affects inbreeding depression in mammalian populations. J Hered 88: 1375-1384.

Barrett SCH, Charlesworth D (1991). Effects of a change in the level of inbreeding on the genetic load. Nature 352: 522-524.

Bowman JC, Falconer DS (1960). Inbreeding depression and heterosis of litter size in mice. Genet Res 1: 262-274.

Bryant EH, Meffert LM, McCommas SA (1990). Fitness rebound in a serially bottlenecked populations of the house fly. Am Nat 136: 542-549.

Byers DL, Waller DM (1999). Do plant populations purge their genetic load? Effects of population size and mating history on inbreeding depression. Ann Rev Ecol Syst 30: 479-513.

Charlesworth B, Charlesworth D (1999). The genetic basis of inbreeding depresion. Genet Res Cambridge 74: 329-340.
Charlesworth D, Charlesworth B (1987). Inbreeding depression and its evolutionary consequences. Ann Rev Ecol Syst 18: 237-268.

Crow JF (1999). The rise and fall of overdominance. Plant Breed Rev 17: 225-257.

Diaz A, Okabe K, Eckenrode CJ, Villani MG, Oconnor BM (2000). Biology, ecology and management of the bulb mites of the genus Rhizoglyphus (Acari: Acaridae). Exp Appl Acarol 24: 85-113.

Fowler K, Whitlock MC (1999). The variance in inbreeding depression and the recovery of fitness in bottlenecked populations. Proc R Soc London B 266: 2061-2066.

Frankham R (1995). Conservation genetics. Ann Rev Genet 29: 305-327.

Garcia N, Lopez-Fanjul C, Garcia-Dorado A (1994). The genetics and viability in Drosophila melanogaster: effects of inbreeding and artificial selection. Evolution 48: 1277-1285.

Gerson U, Capua S, Thorens D (1983). Life history and life tables of Rhizoglyphus robini Cleparede (Acari: Astigmata: Acaridae). Acarologia 24: 439-448.

Glass GV, Peckham PD, Sanders JR (1972). Consequences of failure to meet assumptions underlying the fixed effects analysis of variance and covariance. Rev Educ Res 42: 239288.

Hedrick PW (1994). Purging inbreeding depression and the probability of extinction: full-sib mating. Heredity 73: 363-372.

Heinemann R, Hughes RD (1970). Reproduction, reproductive organs, and meiosis in bisexual non-parthenogenetic mite Caloglyphus mycophagus, with reference to oocyte degeneration in virgins (Sarcoptiformes: Acaridae). J Morphol 130: 93-102.

Houle D (1998). How should we explain variation in genetic variation of traits. Genetica 102/103: 241-253.

Houle D, Hughes KA, Assimacopoulos S, Charlesworth B (1997). The effects of spontaneous mutation on quantitative traits. II. Dominance of mutations with effects on life-history traits. Gen Res Cambridge 70: 27-34.

Houle D, Kondrashov AS (2002). Coevolution of costly mate choice and condition-dependent display of good genes. Proc $R$ Soc London B 269: 97-104.

Karkkainen K, Kuittinen H, van Treuren R, Vogl C, Oikarinen S, Savolainen O (1999). Genetic basis of inbreeding depression in Arabis petrea. Evolution 53: 1354-1365.

Keller LF, Waller DM (2002). Inbreeding effects in wild populations. Trends Ecol Evol 17: 230-241.

Konior M, Radwan J, Kołodziejczyk M (2001). Polyandry increases offspring fecundity in the bulb mite. Evolution $\mathbf{5 5}$ 1893-1896.

Latter BDH, Mulley JC, Reid D, Pascoe L (1995). Reduced genetic load revealed by slow inbreeding in Drosophila melanogaster. Genetics 139: 287-297.

Lynch CB (1977). Inbreeding effects upon animals derived from a wild population of Mus musculus. Evolution 31: 526-537.

Lynch M, Walsh B (1998). Genetics and Analysis of Quantitative Traits. Sinauer Associates: Sunderland, MA.

Oliver Jr JH (1971). Parthenogenesis in mites and ticks. Am Zool 11: $283-299$.

Oliver Jr JH (1977). Cytogenetics of mites and ticks. Ann Rev Entomol 22: 407-429.

Radwan J (1997). Sperm precedence in the bulb mite, Rhizoglyphus robini: context-dependent variation. Ethol Ecol Evol 9: 373-383.

Rice WR, Gaines SD (1994). Extending nondirectional heterogeneity tests to evaluate simply ordered alternative hypotheses. Proc Natl Acad Sci USA 91: 225-225.

Roff DA (1998). Evolutionary Quantitative Genetics. Chapman \& Hall: New York.

Roff DA (2002). Inbreeding depression: tests of the overdominance and partial dominance hypotheses. Evolution $\mathbf{5 6}$ 768-775. 
Shabalina SA, Yampolsky LY, Kondrashov AS (1997). Rapid decline of fitness in panmictic populations of Drosophila melanogaster maintained under relaxed natural selection. Proc Natl Acad Sci USA 94: 13034-13039.

Sittman K, Abplanalp HA, Fisher RA (1966). Inbreeding depression in Japanese quail. Genetics 54: 371-379.

Tempelton AR, Read B (1983). Factors eliminating inbreeding depression in a captive herd of Speke's gazelle. Zoo Biol 3: 177-199.

Wang J (2000). Effects of population structures and selection strategies on the purging of inbreeding depression due to deleterious mutations. Genet Res Cambridge 76: 75-89.

Wang J, Hill WG, Charlesworth D, Charlesworth B (1999). Dynamics of inbreeding depression due to deleterious mutations in small populations: mutation parameters and inbreeding rate. Genet Res Cambridge 74: 165-178.

Willis JH (1999). The role of genes of large effect on inbreeding depression in Mimulus guttatus. Evolution 53: 1678-1691.

Woodring JP (1969). Observations on the biology of six species of acarid mites. Ann Entomol Soc Am 62: 102-108. 